\title{
Dose-related treatment outcomes in South African patients prescribed clofazimine for drug-resistant tuberculosis
}

\author{
N Misra, ${ }^{1}$ BPharm, MMedSc (Clinical Pharmacology), PhD; N Padayatchi, ${ }^{2} \mathrm{MB}$ ChB, $\mathrm{PhD}$; \\ P Naidoo, ${ }^{1}$ BPharm, MMedSc (Pharmacology), PhD

\begin{abstract}
${ }^{1}$ Discipline of Pharmaceutical Sciences, School of Health Sciences, College of Health Sciences, University of KwaZulu-Natal, Durban, South Africa ${ }^{2}$ HIV-TB Pathogenesis and Treatment Research Unit, Centre for the AIDS Programme of Research in South Africa (CAPRISA), South African
\end{abstract} \\ Medical Research Council, Durban, South Africa
}

Corresponding author: N Misra (nirupa.misra@kznhealth.gov.za)

\begin{abstract}
Background. Optimal drug levels and minimal toxicity are critical factors in improving treatment outcomes for patients' prescribed new and repurposed medicine for drug-resistant (DR) tuberculosis (TB). The optimal dose of clofazimine (CFZ), a repurposed medicine for DR-TB, that is safe and effective in the South African (SA) population is unknown.

Objectives. To report on dose-related final treatment outcomes in patients receiving CFZ plus a background regimen for DR-TB.

Methods. In a retrospective review of patient folders from 2012 to 2014, treatment outcomes documented for patients receiving high$(\geq 200 \mathrm{mg})$ and low-dose $(100 \mathrm{mg}) \mathrm{CFZ}$ in a centralised DR-TB hospital in KwaZulu-Natal Province, SA, were investigated for an association between dose-weight interactions and outcomes.

Results. A total of 600 patients were included, of whom $169(28.2 \%)$ received $100 \mathrm{mg}$. Of these, 87 (51.5\%) weighed $<50 \mathrm{~kg}$ and $82(48.5 \%)$ $\geq 50 \mathrm{~kg}$. Four hundred and thirty-one $(71.8 \%)$ received $\geq 200 \mathrm{mg}$, of whom $41(9.5 \%)$ were $<50 \mathrm{~kg}$ and $390(90.5 \%) \geq 50 \mathrm{~kg}$. Overall $77.2 \%$ were HIV-positive, with $93.95 \%$ on antiretroviral medicine. The majority of patients presented with extremely drug-resistant $\mathrm{TB}(55.3 \%)$. Forty-seven and a half percent of patients received a standardised background regimen, and $52.5 \%$ received an individualised regimen containing a new or repurposed medicine including CFZ. On multivariate analysis, adjusting for age, gender, HIV status and concomitant antiretrovirals, previous TB history, type of TB and background regimen, patients $\geq 50 \mathrm{~kg}$ prescribed $100 \mathrm{mg}$ CFZ were $60 \%$ less likely to have a successful outcome (adjusted odds ratio (OR) $0.4 ; 95 \%$ confidence interval (CI) $0.2-0.8 ; p=0.009$ ) compared with patients $<50 \mathrm{~kg}$ receiving $100 \mathrm{mg}$ CFZ. Patients $<50 \mathrm{~kg}$ who received $\geq 200 \mathrm{mg}$ were $40 \%$ less likely to have a successful treatment outcome (adjusted OR 0.6 , $p=0.3$ ), and were found to have a higher risk of adverse events than patients $<50 \mathrm{~kg}$ receiving $100 \mathrm{mg} \mathrm{CFZ}(82.9 \% \mathrm{v} .65 .5 \%)$.

Conclusions. Dose-weight interaction plays a role in the odds of a successful outcome. There is an association between dose-weight interactions, outcomes and adverse events. Weight-based dosing in patients $<50 \mathrm{~kg}$ and $\geq 50 \mathrm{~kg}$ must be considered to achieve optimal treatment outcomes and reduce adverse events. Active drug safety monitoring must be implemented as a package of care for patients receiving CFZ as part of a DR-TB treatment regimen.
\end{abstract}

S Afr Med J 2021;111(1):61-67. https://doi.org/10.7196/SAMJ.2020.v11111.14605

Tuberculosis (TB) is the ninth-leading cause of death worldwide, with the global TB 2019 report placing South Africa (SA) among the six high TB burden countries. Globally, treatment success rates (cured and completed treatment) for multidrug-resistant TB (MDR-TB) was 56\%, and for extensively drug-resistant TB (XDR-TB) $39 \%$ in the 2016 cohort. ${ }^{[1]}$ Despite major investments in medicine, diagnostics and programmatic management, low treatment success rates, high failure and loss to follow-up rates are reported for DR-TB globally and in SA.

Historically, treatment for DR-TB has been lengthy (18 24 months), with an injection given for 6 - 9 months in combination with oral medicine. ${ }^{[2-4]}$ In 2016, The World Health Organization (WHO) published updated guidelines for the management of MDRTB that reduced the duration of treatment to $9-11$ months in selected patients diagnosed with MDR-TB. The long regimen is still recommended in patients with pre-XDR-TB and XDR-TB. Clofazimine (CFZ), previously classified as a group 5 medicine and recommended for use in patients with pre-XDR-TB or XDR-TB, has now been reclassified as a core medicine to treat MDR-TB in a novel short-course regimen. ${ }^{[5]}$

The SA DR-TB management guidelines of 2011 (updated 2013) included the use of CFZ for patients with pre-XDR and XDR-TB at high doses $(200 \mathrm{mg}<50 \mathrm{~kg}$ and $300 \mathrm{mg}$ in patients $\geq 50 \mathrm{~kg}$ for 18 24 months) ${ }^{[4]}$ This is despite there being no conclusive evidence on the safety and efficacy of CFZ at high doses for long periods of time. Recently published WHO guidelines (2019) recommend CFZ at a dose of $100 \mathrm{mg}$ daily, despite paucity of data on the optimal dose of $\mathrm{CFZ}$ and the fact that the need for a loading dose is listed as a research priority by the Guideline Development Committee..$^{[5]}$

Several studies have been done to assess the safety and efficacy of CFZ for DR-TB, with promising results for this repurposed medicine, previously used only for leprosy, and which is not registered for TB. ${ }^{[7-10]}$ However, most of these observational studies had small sample sizes, with a common limitation being the absence of dose-related efficacy data. Tang et al., ${ }^{[7]}$ in a multicentre, randomised controlled trial, reported that in patients who received CFZ $100 \mathrm{mg}$ daily as part of a DR-TB treatment regimen, the treatment success rate was $73.6 \%$, higher than that in the control group $(53.8 \%$; $p=0.035)$. Although the study reported good tolerance and low toxicity of CFZ, a limitation identified was the variable doses of CFZ used in other studies, and it was recommended that the proper dosage of CFZ for treatment of MDR-TB should be further investigated. ${ }^{[7]}$

This was identified as a gap in knowledge to optimise the safety and efficacy of CFZ for DR-TB. Various systematic reviews and 
meta-analyses assessing outcomes of a CFZ-containing DR-TB regimen have consistently shown that $\mathrm{CFZ}$ has potential to improve treatment outcomes. However, there are insufficient data on dosage and duration of therapy for CFZ to be meaningfully assessed, and the optimal dose of CFZ and duration of use require further investigation. ${ }^{[6,7,9,10]}$ Hwang et al. ${ }^{[9]}$ reviewed a comprehensive body of peer-reviewed literature and policy guidance relating to the safety, use, cost and availability of clofazimine in clinical practice, and concluded that clofazimine was associated with a risk for adverse drug reactions comparable with that of first-line TB treatment, which could be managed under programmatic conditions.

The efficacy and safety of a clofazimine-containing regimen in MDR-TB at a dose of $100 \mathrm{mg}$ was demonstrated by Tang et al..$^{[7]}$ in a multicentre randomised controlled trial.

Clofazimine appeared to be associated with a lower incidence of serious adverse effects than other second-line therapeutics, and could potentially be considered as an additional therapeutic agent for the treatment of MDR-TB. A reported limitation of their study was the absence of comparing the different doses of CFZ to identify the optimum safe dose, irrespective of whether a better or worse result would have been obtained with higher doses. Tang et al. ${ }^{[7]}$ pointed out that while most researchers used $100 \mathrm{mg}$ once daily, there has been no comparison between different dosages of CFZ.

The study noted that the daily dose of $100 \mathrm{mg}$, based solely on expert opinion by a WHO consultation group, required further investigation to establish the proper dosage of CFZ for treatment of MDR-TB. ${ }^{[7]}$ The literature further highlighted that studies reporting on the dose of CFZ used and researched did not investigate the most effective dose for the treatment of DR-TB in SA adult patients. Studies on the optimum dose of CFZ that is safe and effective for the treatment of DR-TB in the SA setting or globally are not available..$^{[6,7,10,11]}$

Numerous studies have shown that absorption of orally administered CFZ varies considerably (45\% - 62\%) depending on whether the drug is taken with or without food. ${ }^{[10-14]}$ There is also inter-patient variation in the bioavailability of CFZ, and the limited activity of clofazimine against Mycobacterium tuberculosis in humans may be due to inadequate peak drug concentrations or an insufficient total dose as a result of low oral bioavailability and gastric intolerance. Hence administering the correct dose of CFZ is critical to avoid further resistance from emerging.

The present study attempts to compare dose-related efficacy of CFZ in SA patients receiving high- and low-dose CFZ as part of a treatment regimen for DR-TB.

\section{Objectives}

To determine the association between the dose of CFZ prescribed at different weight bands and final treatment outcomes in the SA population.

\section{Methods}

\section{Study design, patients and procedures}

This was a retrospective cohort study of adult patients diagnosed with DR-TB in KwaZulu-Natal (KZN) Province, SA, treated with either a high-dose clofazimine or low-dose clofazimine-containing DR-TB treatment regimen between 2012 and 2014. The primary outcome measure was final treatment outcomes at 24 months. Multivariate logistic regression compared successful outcomes with unsuccessful outcomes. Risk factors for unsuccessful outcomes were identified.

The frequency and types of adverse events, defined as a documented side-effect in the patients' clinical folder, irrespective of grading or intervention, which affected a body system in a negative way, were investigated and analysed in patients receiving $100 \mathrm{mg}$ and $\geq 200 \mathrm{mg}$ CFZ. Multivariate logistic regression compared the risk of any adverse events in each cohort, taking into account possible confounders that could have contributed to the adverse event.

During the study period, the pharmacy database showed 1018 patients listed as receiving CFZ, of which 365 entries did not have a traceable DR-TB clinical folder at the centralised unit. These entries were linked to patients who were randomly down-referred to a satellite site in eThekwini for management. A total of 600 adult patients were managed at the centralised DR-TB unit, had traceable DR-TB numbers that enabled file retrieval and were included in the study, as per inclusion criteria (Fig. 1).

Patient clinical folders were retrieved and data extracted using manual data collection forms. Information on the starting dose of CFZ, demographics, HIV status and antiretroviral status, previous TB history, drug exposure, baseline tests, drug resistance patterns, background regimen and treatment outcomes at 24 months was collected. Missing information in patients' folders was sourced from the National Health Laboratory Services database and Tier.net, where applicable. All data extracted and captured were verified by the principal investigator.

\section{Treatment outcome definitions}

The primary outcome measure was final treatment outcomes at 24 months as defined by the WHO in 2008. ${ }^{[2]}$

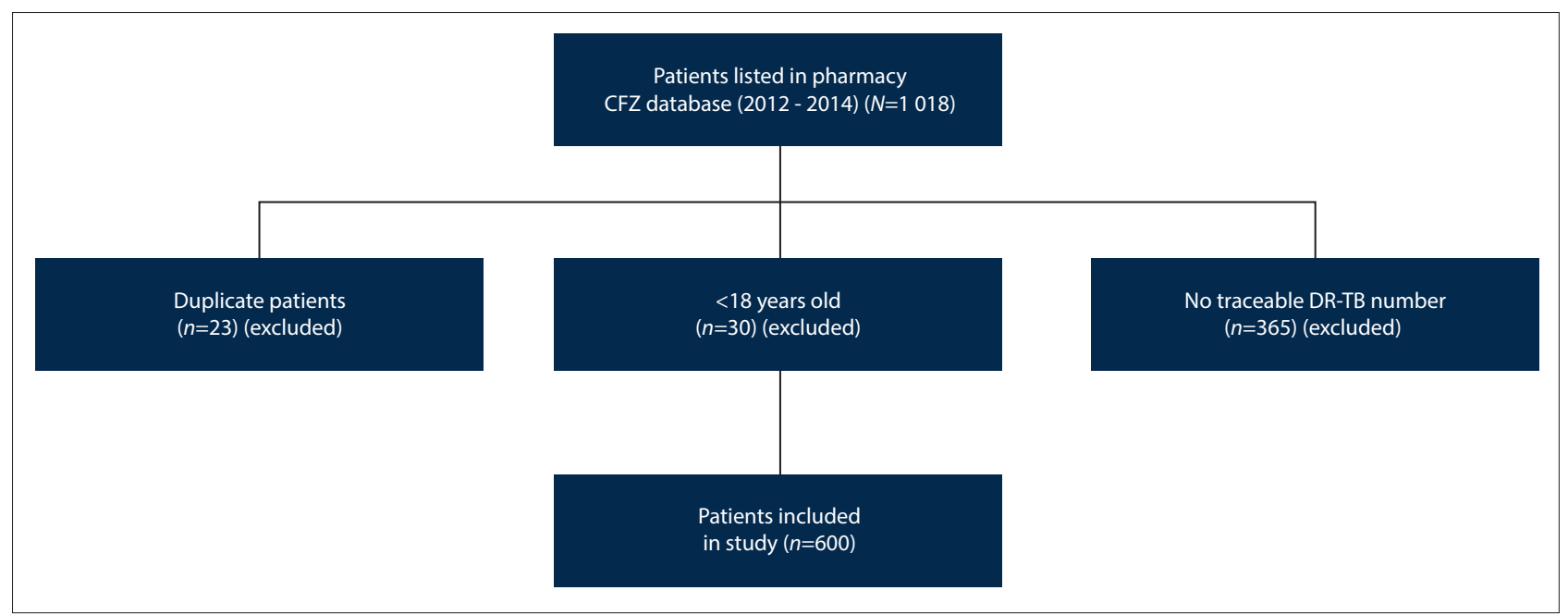

Fig. 1. Patient enrolment $(C F Z=$ clofazimine; $D R-T B=$ drug-resistant tuberculosis. $)$ 
'Cured' referred to a patient who had completed treatment according to programme protocol and had at least 5 consecutive negative cultures from samples collected at least 30 days apart in the final 12 months of treatment. 'Treatment completed' referred to a patient who had completed treatment according to programme protocol but did not meet the definition for cure because of lack of bacteriological results (i.e. $<5$ cultures were performed in the final 12 months of treatment). Patients who were cured and/or completed treatment were categorised as having a successful treatment outcome for analysis.

An outcome of 'died' referred to a patient who died for any reason during the course of MDR-TB treatment. A patient was considered to have 'failed' if $\geq 2$ of the 5 cultures recorded in the final 12 months of therapy were positive, or if any one of the final 3 cultures was positive. Treatment was also considered to have failed when a clinical decision was made to terminate treatment early because of poor clinical or radiological response or adverse events. A patient whose treatment was interrupted for 2 or more consecutive months for any reason without medical approval was classified as defaulted. Patients with an outcome of died, failed or defaulted were classified as having unsuccessful treatment outcomes for analysis. The current definition of default has been changed to loss to treatment follow-up.

Adverse events documented in the clinical folders were categorised according to the different body systems, such as the muscular system, gastrointestinal tract, eyes, skin, central nervous system, ears, feet, kidney, heart and liver. All adverse events noted in the clinical folders were recorded and analysed irrespective of whether CFZ was noted as the suspected agent.

\section{Statistical analysis}

Patient clinical records were reviewed to collect patient-related demographic, clinical, pharmaceutical and laboratory data. Entry of data was done on an Excel (Microsoft, USA) spreadsheet, and discordances were identified and resolved through verification with the original paper records by the principal investigator. The validated database was analysed using Stata (StataCorp, USA) version 13.0.

Patients were stratified into two groups: those receiving $100 \mathrm{mg}$ CFZ (low-dose group) and those receiving doses $\geq 200 \mathrm{mg}$ (high-dose group). Logistic regression was used to assess the effect of risk factors on successful v. unsuccessful outcomes, adjusted for confounders. Univariate and multivariate statistics, 95\% confidence intervals and $p$-values were used where appropriate to analyse the data. Statistical significance was set at $p<0.05$.

\section{Ethical considerations}

Ethics approval was obtained from the University of KZN Biomedical Research Ethics Committee to conduct the study (ref. no. BE466/14). The study was also approved by the KZN Health Research Unit.

\section{Results}

Of the 1018 patients registered on the pharmacy CFZ database between 2012 and 2014, 23 entries were identified as duplicate patients, 30 were $<18$ years old and 365 were managed at a satellite down-referral unit and were excluded as per exclusion criteria. A total of 600 patients met the study inclusion criteria of $\geq 18$ years, bacteriologically confirmed rifampicin-resistant TB and availability of a clinical folder with treatment outcomes and dose of CFZ recorded. Patients were grouped into those receiving low-dose CFZ $(100 \mathrm{mg})$ and high-dose CFZ ( $\geq 200 \mathrm{mg})$.

The baseline demographic and clinical characteristics of patients receiving low- and high-dose CFZ are summarised in Table 1.
There were no differences in age, gender, HIV status and whether on antiretroviral (ARV) therapy between the two groups. Differences were observed in the weight distribution of patients receiving low- and high-dose CFZ. A significantly larger number of patients weighed $\geq 50 \mathrm{~kg}$ (78.7\%), of whom 390 (90.5\%) received $\geq 200 \mathrm{mg}$ CFZ and 82 (48.5\%) received $100 \mathrm{mg} \mathrm{CFZ.}$

Overall, $44.5 \%$ of patients had had a previous episode of DR-TB, with $51.5 \%$ receiving high-dose CFZ and $26.6 \%$ receiving a low dose $(p<0.01)$. A significantly high number of patients presented with XDR-TB (55.3\%), with $44.4 \%$ of these receiving low-dose CFZ and $59.6 \%$ receiving high-dose CFZ.

Univariate analysis of gender indicated that females were 1.39 times more likely to have a successful treatment outcome than males. This was confirmed on multivariate analysis, with females showing 1.5 times greater likelihood of having a successful treatment outcome than males (adjusted odds ratio (OR) 1.5, $p=0.03$ ) (Table 2).

The impact of concurrent first- or second-line ARVs could not be determined owing to missing information on the type of ARVs prescribed.

Patients with a previous episode of TB (DR- or drug-sensitive TB) were $40 \%$ less likely to have a successful treatment outcome than patients with no previous TB episode $(p=0.01)$.

Among all patients who had a successful treatment outcome $(n=279)$, the background regimen of $148(47.0 \%)$ included new or repurposed medicine, and $167 / 321(53.0 \%)$ patients with an unsuccessful treatment outcome were found to include new or repurposed medicine excluding CFZ. There was no difference in the likelihood of overall success among the two groups (OR 1,95\% confidence interval (CI) 0.8 - 1.4).

Overall treatment outcomes in patients receiving high- and low-dose CFZ at initiation are shown in Table 3, and dose-weight interaction is further explored in Table 4.

The odds of success in patients weighing $\geq 50 \mathrm{~kg}$ receiving $100 \mathrm{mg}$ CFZ were $60 \%$ lower than in patients $<50 \mathrm{~kg}$ receiving $100 \mathrm{mg}$ CFZ (adjusted OR 0.4, 95\% CI $0.2-0.8, p=0.009$ ), adjusted for variables as listed in Table 4 . This finding was statistically significant.

Patients weighing $<50 \mathrm{~kg}$ who received $\geq 200 \mathrm{mg}$ CFZ were also $40 \%$ less likely to have a successful treatment outcome than patients $<50 \mathrm{~kg}$ who received $100 \mathrm{mg}$ CFZ (adjusted OR 0.6, 95\% CI $0.3-1.4, p=0.3$ ). This result is not statistically significant, but may be explained by Table 5 .

Similar results were found when the reference category was changed to $\geq 200 \mathrm{mg}$ and $\geq 50 \mathrm{~kg}$, with patients $<50 \mathrm{~kg}$ receiving $\geq 200 \mathrm{mg}$ CFZ having $51 \%$ lower chance of a successful treatment outcome (adjusted OR $0.49,95 \%$ CI $0.3-0.8, p=0.008$ ).

This dose-weight interaction was explored further by assessing the impact of dose and weight on the presentation of adverse events, as shown in Table 5 .

Any adverse event refers to an untoward event documented in the patient's clinical folder, irrespective of a causal relationship with CFZ.

Dose-weight interactions impact on the presentation of adverse events. Patients $<50 \mathrm{~kg}$ who received $\geq 200 \mathrm{mg} \mathrm{CFZ}$ were 2.57 times more likely to experience an adverse event than patients $<50 \mathrm{~kg}$ who received $100 \mathrm{mg}$ CFZ (adjusted OR 2.57, 95\% CI 1.02 - 6.50, $p=0.046$ ).

\section{Discussion}

This is the first study that we are aware of that compares drugresistant treatment outcomes in SA patients receiving varying doses of CFZ, together with a background regimen selected on the basis of the individual patient's drug-resistance patterns and expert opinion. Although many studies have been conducted to assess the safety and efficacy of CFZ together with other second-line TB medicine, the inclusion of data on dose of CFZ administered and related outcomes has been minimal. ${ }^{[6,7,10,11]}$ 


\begin{tabular}{|c|c|c|c|c|}
\hline Variable & $100 \mathrm{mg}, n(\%)^{\star}$ & $\geq 200 \mathrm{mg}, n(\%)^{\star}$ & Total, $n(\%)^{\star}$ & $p$-value \\
\hline Sample size & $169(28.2)$ & $431(71.8)$ & $600(100)$ & \\
\hline Age, median (IQR) & $34(18-77)$ & $34(18-80)$ & $34(18-80)$ & 0.4 \\
\hline \multicolumn{5}{|l|}{ Gender } \\
\hline Male & $84(49.7)$ & $209(48.5)$ & $293(48.8)$ & \multirow[t]{2}{*}{0.8} \\
\hline Female & $85(50.3)$ & $222(51.5)$ & $307(51.2)$ & \\
\hline \multicolumn{5}{|l|}{ Weight } \\
\hline$<50 \mathrm{~kg}$ & $87(51.5)$ & $41(9.5)$ & $128(21.3)$ & \multirow[t]{2}{*}{$<0.001$} \\
\hline$\geq 50 \mathrm{~kg}$ & $82(48.5)$ & $390(90.5)$ & $472(78.7)$ & \\
\hline \multicolumn{5}{|l|}{ HIV status } \\
\hline Positive & $133(79.2)$ & $330(76.7)$ & $463(77.2)$ & \multirow[t]{2}{*}{0.5} \\
\hline Negative & $35(20.8)$ & $100(23.3)$ & $137(22.8)$ & \\
\hline HIV-positive on ART & $123(92.5)$ & $312(94.5)$ & $435(93.95)$ & 0.4 \\
\hline \multicolumn{5}{|l|}{ Previous TB History } \\
\hline Previous drug-sensitive TB & $77(45.6)$ & $150(34.8)$ & $227(37.8)$ & $<0.001$ \\
\hline Previous DR-TB & $45(26.6)$ & $222(51.5)$ & $267(44.5)$ & \multirow[t]{2}{*}{$<0.001$} \\
\hline No previous history of $\mathrm{TB}$ & $47(27.8)$ & $59(13.7)$ & $106(17.7)$ & \\
\hline \multicolumn{5}{|l|}{ Treatment initiation } \\
\hline Inpatient & $95(56.2)$ & $282(65.4)$ & $377(62.8)$ & \multirow[t]{2}{*}{0.04} \\
\hline Outpatient & $74(43.8)$ & $149(34.6)$ & $223(37.2)$ & \\
\hline \multicolumn{5}{|l|}{ Background regimen type } \\
\hline Standardised background regimen plus CFZ & $65(38.5)$ & $220(51.0)$ & $285(47.5)$ & \multirow[t]{2}{*}{$<0.001$} \\
\hline $\begin{array}{l}\text { Individualised regimen including new and repurposed medicine } \\
\text { (BDQ, LZD, imipenem) }\end{array}$ & $104(61.5)$ & $211(49.0)$ & $315(52.5)$ & \\
\hline \multicolumn{5}{|l|}{ Type of TB } \\
\hline Rifampicin-resistant TB & $39(23.1)$ & $56(13.0)$ & $95(15.8)$ & \multirow[t]{3}{*}{$<0.004$} \\
\hline MDR-TB & $22(13.0)$ & $57(13.2)$ & $79(13.2)$ & \\
\hline Pre-XDR-TB & $28(16.6)$ & $54(12.5)$ & $82(13.7)$ & \\
\hline XDR-TB & $75(44.4)$ & $257(59.6)$ & $332(55.3)$ & \multirow[t]{2}{*}{0.001} \\
\hline Unknown & $5(3.0)$ & $7(1.6)$ & $12(2.0)$ & \\
\hline
\end{tabular}

The major findings of this study indicate that dose-weight interactions have a significant impact on the odds of a successful outcome. When stratified according to dose-weight categories, patients in the high weight band ( $\geq 50 \mathrm{~kg}$ ) who received $100 \mathrm{mg}$ CFZ had a $60 \%$ lower chance of a successful outcome than patients $<50 \mathrm{~kg}$ receiving $100 \mathrm{mg}$ CFZ (adjusted OR 0.4, 95\% CI $0.2-0.8, p=0.009$ ).

Following extensive review of the literature, the guideline development committee of the WHO includes CFZ as a core medicine to treat DR-TB in the 2019 updated guidelines for the management of DR-TB, while acknowledging several gaps in current knowledge about critical areas. No decision could be made on whether a loading dose of CFZ was required, owing to lack of data. ${ }^{[5]}$ This study moves the evidence towards loading doses being required, especially in weight bands $\geq 50 \mathrm{~kg}$.

Low starting dose of CFZ in high weight-band patients $(\geq 50 \mathrm{~kg}$ ) was found to be a negative variable for a successful treatment outcome. This finding is supported by studies that reviewed the pharmacokinetics and pharmacodynamics of CFZ, and recommend weight-based dosing to achieve the required minimum inhibitory concentrations. ${ }^{[12,16]}$ The findings of the present study are further supported by Schaad-Lanyi et al., ${ }^{[15]}$ who compared the pharmacokinetics of CFZ in 12 healthy volunteers following a single dose of $200 \mathrm{mg}$ and multiple doses of $50 \mathrm{mg}$ daily, and recommended weightbased dosing. ${ }^{[15]}$
Higher starting doses of CFZ (up to $300 \mathrm{mg}$ ) have been supported by previous WHO programmatic guidelines for the management of DR-TB. ${ }^{[2]}$ A companion handbook published in 2014 also supported the use of CFZ $200-300 \mathrm{mg}$ daily for 2 months followed by $100 \mathrm{mg}$ daily, despite concerns about overlapping cardiotoxicity with bedaquiline ${ }^{[4]}$ Weight-based dosing of CFZ was also recommended in the SA MDR-TB guidelines 2011 (updated in 2013). ${ }^{[4]}$ New SA DR-TB treatment guidelines published in November 2019 recommend $100 \mathrm{mg}$ CFZ across weight bands, despite there being poor evidence on the optimal dose of CFZ that is effective and safe. ${ }^{[17]}$

Inter-patient variability ( $45 \%-62 \%$ variability in absorption) and the effects of food on absorption were demonstrated by Cholo et al., ${ }^{[11]}$ and it was proposed that the limited activity of CFZ against Mycobacterium tuberculosis in humans may be due to inadequate peak drug concentrations, or an insufficient total dose as a result of low oral bioavailability. This is aligned to our findings that low-dose CFZ is a negative variable for a successful treatment outcome in patients weighing $\geq 50 \mathrm{~kg}$.

Padayatchi et al.$^{[6]}$ found that there was a delay in culture conversion in patients receiving CFZ, which may be due to extensive binding and time required to accumulate in tissues. In this study, CFZ was used at high doses of $\geq 200 \mathrm{mg}$ daily, with patients receiving a CFZcontaining regimen showing better treatment outcomes. This finding further supports the use of higher starting doses of CFZ, together 


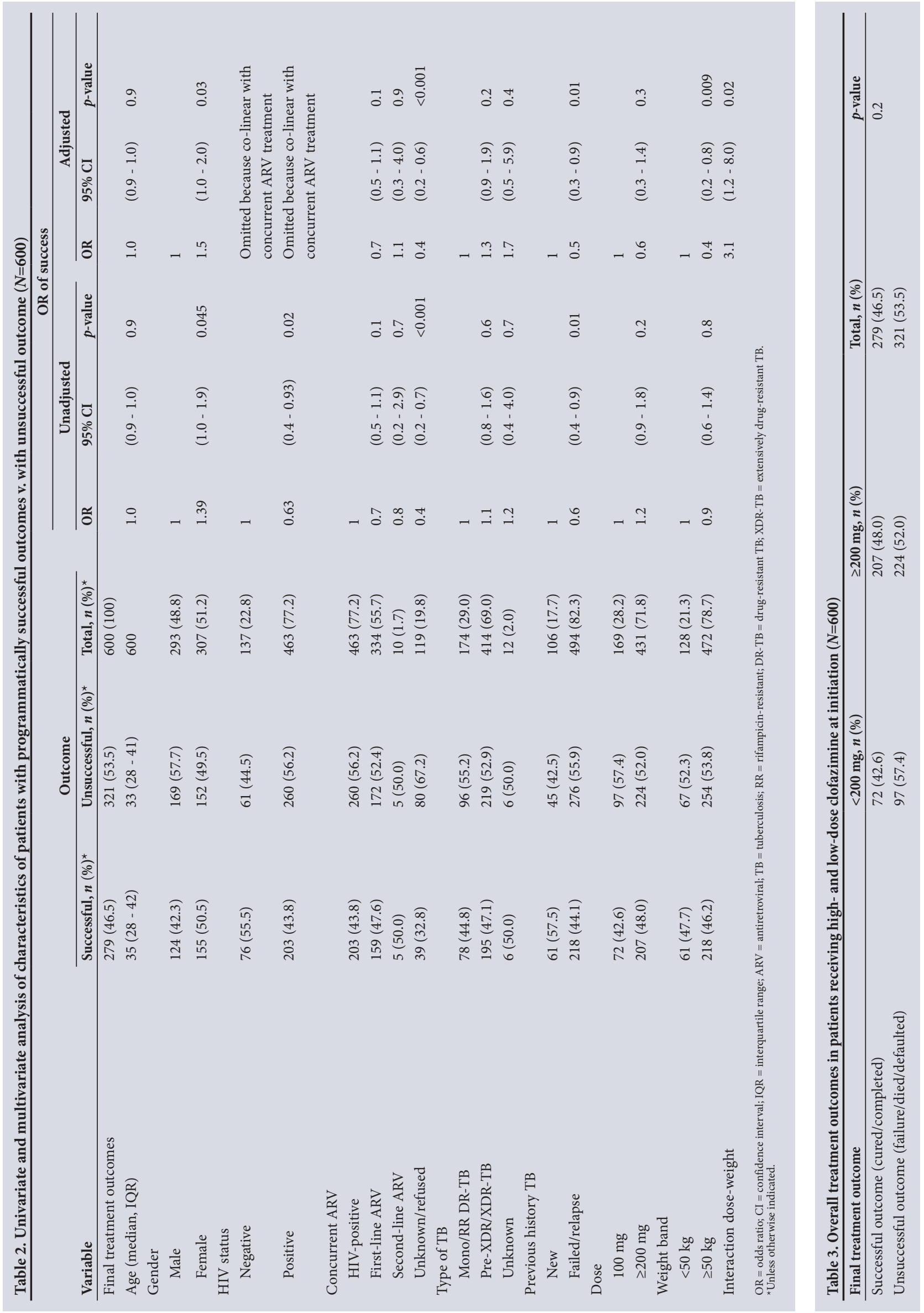



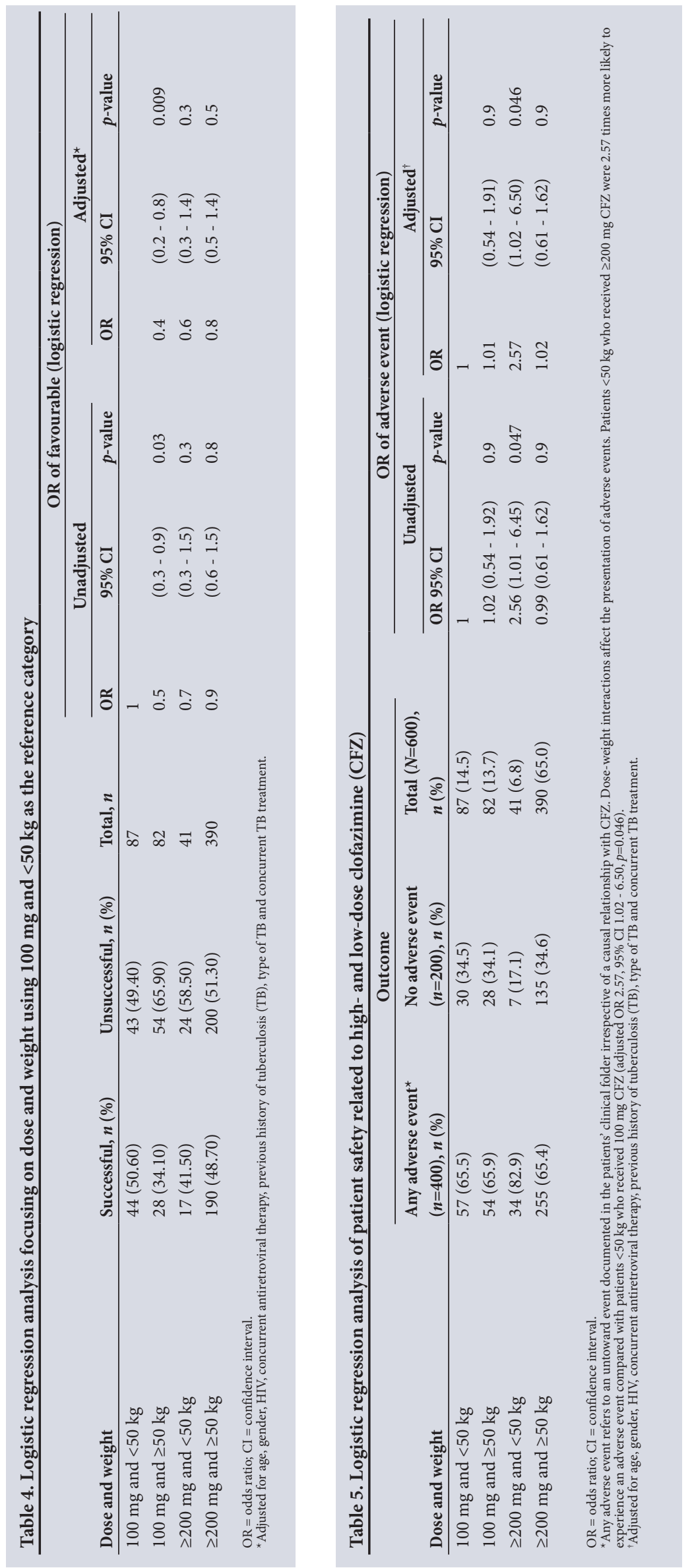

with a suitable background regimen to achieve better treatment outcomes. In a study by $\mathrm{Xu}$ et al. ${ }^{[18]}$ it was noted that low-dose CFZ (100 mg) once daily was well tolerated and appeared to be effective when given with a suitable background regimen. However, the sample population was small and conclusions could not be made on the optimal dose of CFZ that maximises efficacy while minimising toxicity. ${ }^{[18]}$

Recent WHO treatment guidelines for DR-TB recommend the use of CFZ as a core drug in a short-course DR-TB treatment regimen at a dose of $100 \mathrm{mg}$ daily. ${ }^{[19]}$ This recommendation is based mainly on observational studies and not randomised controlled clinical trials. ${ }^{[8]}$ No studies were available until now that compare treatment outcomes in patients receiving high- and lowdose CFZ. The present study's findings that doseweight interactions are a significant confounder for successful treatment outcomes must be considered when revising the current standard treatment guidelines for managing patients with DR-TB in SA.

Safety data indicate that there is also an association between the dose of CFZ prescribed at different weight bands and the odds of an adverse event occurring. Low-weight patients $(<50 \mathrm{~kg})$ who received high doses of CFZ ( $\geq 200 \mathrm{mg})$ experienced more side-effects than patients $<50 \mathrm{~kg}$ who received $100 \mathrm{mg}$ CFZ (82.9\% v. 65.5\%). This supports the recommendation of lower doses of CFZ being used in low weight-band patients.

The present study also found that female patients were more likely to have had a successful treatment outcome than their male counterparts (adjusted OR 1.5, 95\% CI $1.0-2.0, p=0.03$ ), adjusting for all variables. This finding, however, was not supported by the Bangladesh study ${ }^{[8]}$ that reported that females (v. males) had an increased risk of an unfavourable treatment outcome. Further work needs to be done in assessing the impact of gender on treatment outcomes.

It was noted that patients co-infected with HIV were less likely to have a successful treatment outcome than HIV-negative patients, which was supported by a previous study conducted in KZN in patients co-infected with XDR-TB and HIV. ${ }^{[6]}$ The impact of first-line or second-line ARVs on treatment outcomes could not be assessed owing to missing data, and further research is required. It is also recommended that $\mathrm{TB} / \mathrm{HIV}$ management be integrated with one prescriber for both conditions, in order to monitor drug-drug interactions and overlapping toxicities of DR-TB medicine and ARVs that may impact on treatment outcomes.

The studies above all indicate that CFZ is a drug that will likely continue to play a significant role in the treatment of TB (and in fact there are trials planning to assess its use in treatment shortening for drug-susceptible TB), and highlight the importance of finding an optimal dosing strategy. 
The present study provides new data on dose-related outcomes of patients initiated on CFZ as part of a DR-TB regimen, and should be considered in the use of CFZ in the SA population.

\section{Study limitations}

The study has several limitations. CFZ was used as part of a treatment regimen that included other second-line DR-TB medication, thus outcomes (treatment success and adverse events) cannot be definitively ascribed to CFZ. However, both groups received a similar background regimen, apart from the dose of CFZ that was being investigated.

The retrospective nature of the study is not optimal, however. As a result of non-evidence-based changes in prescribing habits due to anecdotal concerns regarding toxicity, no patients in cohorts after 2014 received doses of CFZ $\geq 200 \mathrm{mg}$ in KZN. A retrospective study that included SA patients who received highand low-dose CFZ within the different weight bands during the study period provided an opportunity to collect information on the optimal dose of CFZ that was effective and safe in the SA population.

The exclusion of patients who were down-referred to the satellite facility and not included in the study may also be considered a limitation. However, these patients were randomly down-referred based on bed capacity, and would have comprised patients from both study groups. The required sample size was also achieved, which makes the study results valid.

Missing information on the ARV prescribed also is a limitation that must be considered. A further limitation was the fact that adverse events may not have been recorded consistently in the clinical folders; however, this factor will apply similarly across both cohorts. The type of DR-TB and how many episodes of TB the patient had had previously was not very clearly documented. This may also have affected outcomes, and is a limitation.

Despite these limitations, our study adds important knowledge on the subject of the optimal dose of CFZ that is safe and effective for the treatment of DR-TB in a largely HIV-infected SA cohort.

\section{Conclusion}

This study is the only one to our knowledge that compares treatment outcomes in SA patients prescribed varying doses of CFZ stratified across high and low weight bands. Owing to the paucity of data on the optimum dose of CFZ that is safe and effective for the treatment of DR-TB, the authors suggest that the results of this study be taken into consideration, and weight-based dosing of CFZ be adopted.

Based on the findings of this study, high-dose CFZ ( $\geq 200 \mathrm{mg}$ ) is recommended in patients $\geq 50 \mathrm{~kg}$, and low-dose CFZ $(100 \mathrm{mg})$ in patients $<50 \mathrm{~kg}$ owing to the effect of dose-weight interactions on treatment outcomes and adverse events.

Active drug safety monitoring must also be part of the package of care of patients receiving CFZ for DR-TB in SA patients.

Declaration. This publication contributed to the awarding of a $\mathrm{PhD}$ to NM. Acknowledgements. The authors would like to thank the hospital management and all healthcare workers at the study site for their assistance and support. The authors would also like to acknowledge the SA National TB Programme, KZN HIV, AIDS, TB and Sexually Transmitted Infections (HAST) Directorate, healthcare workers and management at the study site. The assistance of Dr Cathy Connolly for statistical guidance and interpretation is greatly appreciated.

Author contributions. NM was responsible for conceptualisation of the study, data collection, analysis and write-up. PN and NP reviewed the article and provided input into the analysis, discussion and conclusions.

Funding. NM was supported by seed funding from the MEPI Grant Implementation Science Traineeship Program funded by the US President's Emergency Plan for AIDS Relief (PEPFAR) through the Fogarty International Center, National Institutes of Health (grant \#D43TW00231) and a further grant awarded by CAPRISA, MRC TB-HIV Pathogenesis Unit, Durban, SA. NM was independent of funders and sponsors.

Conflicts of interest. None.

1. World Health Organization. Global tuberculosis report 2019. Geneva: WHO, 2019. https://www.who. int/tb/publications/global_report (accessed 16 January 2020).

2. World Health Organization. Guidelines for the programmatic management of drug-resistant tuberculosis. Geneva: WHO, 2008. https://apps.who.int/iris/bitstream/handle/10665/43965/9789241547581_eng.pdf; Geneva: WHO, 2008. https://apps.who.int/iris/bitstream/handle/10665/43965/9789241547581_en
jsessionid=B25BD25D49A3E706D9C577039593DE4C?sequence=1 (accessed 4 December 2019).

3. World Health Organization. WHO treatment guidelines for drug-resistant tuberculosis. Geneva: WHO, 2016. https://apps.who.int/iris/bitstream/handle/10665/250125/9789241549639-eng.pdf;jsess WHO, 2016. https://apps.who.int/iris/bitstream/handle/10665/250125/9789241549639-eng
ionid=B25689497A1DF863B5D70F3DBD4841D1? sequence=1 (accessed 7 December 2020).

4. National Department of Health, South Africa. Management of drug resistant tuberculosis: Policy

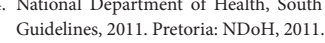

Guidelines, 2011. Pretoria: $\mathrm{NDOH}, 2011$.
World Health Organization. WHO consolidated guidelines on drug-resistant tuberculosis treatment. World Health Organization. WHO consolidated guidelines on drug-resistant tuberculosis treatment.
Geneva: WHO, 2019. https://www.who.int/tb/publications/2019/consolidated-guidelines-drugresistant-TB-treatment/en/ (accessed 16 January 2020).

6. Padayatchi N, Gopal M, Naidoo R, et al. Clofazimine in the treatment of extensively drug-resistant tuberculosis with HIV coinfection in South Africa: A retrospective cohort study. J Antimicrob Chemother 2014;69(11):3103-3107. https://doi.org/10.1093/jac/dku235

7. Tang S, Yao L, Hao X, et al. Clofazimine for the treatment of multidrug-resistant tuberculosis: Prospective, multicenter, randomized controlled study in China. Clin Infect Dis 2015;60(9):1361-1367. https://doi.org/10.1093/cid/civ027

8. Gopal M, Padayatchi N, Metcalfe JZ, O'Donnell MR. Systematic review of clofazimine for the treatment of drug-resistant tuberculosis. Int J Tuberc Lung Dis 2013;17(8):1001-1007. https://doi. org $/ 10.5588$ /ijtld.12.0144

9. Hwang TJ, Dotsenko S, Jafarov A, et al. Safety and availability of clofazimine in the treatment of multidrug and extensively drug-resistant tuberculosis: Analysis of published guidance of multidrug and extensively drug-resistant tuberculosis: Analysis of published guidance
and meta-analysis of cohort studies. BMJ Open 2014;4(1):1-9. https://doi.org/10.1136/ and meta-analysis of

10. Dey T, Brigden G, Cox H, Shubber Z, Cooke G, Ford N. Outcomes of clofazimine for the treatment of drug-resistant tuberculosis: A systematic review and meta-analysis. J Antimicrob Chemother 2013;68(2):284-293. https://doi.org/10.1093/jac/dks389

11. Cholo MC, Steel HC, Fourie PB, Germishuizen WA, Anderson R. Clofazimine: Current status and future prospects. J Antimicrob Chemother 2012;67(2):290-298. http://doi.org/10.1093/jac/dkr444

12. Ma Z, Lienhardt C, Mcllleron H, Nunn AJ, Wang X. Global tuberculosis drug development pipeline: The need and the reality. Lancet 2010;375(9731):2100-2109. http://doi.org/10.1016/S01406736(10)60359-9

13. World Health Organisation. Definitions and reporting framework for tuberculosis - 2013 revision Geneva: WHO: 2013. http://apps.who.int/iris/bitstream/10665/79199/1/9789241505345_eng.pdf (accessed 4 December 2019).

14. Yawalkar SJ, Vischer W. Lamprene (clofazimine) in leprosy. J Lepr 1979;50:135-144. https://doi. org/10.5935/0305-7518.19790020

15. Schaad-Lanyi Z, Dieterle W, Dubois J-PP, Theobald W, Vischer W. Pharmacokinetics of clofazimine Schaad-Lanyi Z, Dieterle W, Dubois J-PP, Theobald W, Vischer W. Pharmacokinetics of clofazimine
in healthy volunteers. Int J Lepr 1987;55(1):9-15. http://www.ncbi.nlm.nih.gov/pubmed/3559339 in healthy volunteers. Int $J$
(accessed 3 December 2019).

16. World Health Organization. Companion handbook to the WHO guidelines for the programmatic management of drug resistant tuberculosis. Geneva: WHO, 2014. http://apps.who.int/iris/ bitstream/10665/75146/1/9789241548441_eng.pdf (accessed 4 December 2019).

17. National Department of Health NTCP, South Africa. Management of rifampicin-resistant tuberculosis A clinical reference guide. Pretoria: NDoH, 2019.

18. Xu HB, Jiang RH, Xiao HP. Clofazimine in the treatment of multidrug-resistant tuberculosis. Clin Microbiol Infect 2012;18(11):1104-1110. https://doi.org/10.1111/j.1469-0691.2011.03716.x

19. World Health Organization. WHO treatment guidelines for drug-resistant tuberculosis: 2016 update. Geneva: WHO, 2016. http://apps.who.int/iris/bitstream/10665/250125/1/9789241549639-eng pdf?ua=1\%0Ahttp://apps.who.int/iris/handle/10665/250125 (accessed 4 December 2019).

20. Aung KJM, Van Deun A, Declercq E, et al. Successful '9-month Bangladesh regimen' for multidrugresistant tuberculosis among over 500 consecutive patients. Int J Tuberc Lung Dis 2014;18(10):1180-
rent 1187. https://doi.org/10.5588/ijtld.14.0100

Accepted 28 April 2020. 